Revue européenne des migrations internationales

vol. 20 - n² | 2004

L'asile politique en Europe depuis l'entre-deux-guerres

\title{
Changements et permanences dans la protection des réfugiés
}

\section{Luc Legoux}

\section{(2) OpenEdition \\ 1 Journals}

\section{Édition électronique}

URL : https://journals.openedition.org/remi/961

DOI : 10.4000/remi.961

ISSN : $1777-5418$

Éditeur

Université de Poitiers

\section{Édition imprimée}

Date de publication : 1 septembre 2004

Pagination : 9-22

ISBN : 2-911627-37-7

ISSN : 0765-0752

Référence électronique

Luc Legoux, "Changements et permanences dans la protection des réfugiés ", Revue européenne des migrations internationales [En ligne], vol. 20 - $n^{\circ} 2$ | 2004, mis en ligne le 25 septembre 2008, consulté le 14 avril 2022. URL : http://journals.openedition.org/remi/961 ; DOI : https://doi.org/10.4000/remi.961

Ce document a été généré automatiquement le 14 avril 2022.

(c) Université de Poitiers 


\title{
Changements et permanences dans la protection des réfugiés
}

\author{
Luc Legoux
}

1 La crise de l'asile, perceptible dès la fin des années quatre-vingt, a été très longue à mûrir et elle n'est analysée comme un changement de système que depuis quelques années. Il faut dire que si le régime fondé sur la convention de Genève de 1951 a été progressivement démantelé, la convention de Genève elle-même n'a jamais été officiellement dénoncée. Le lien très fort entre cette convention et la défense des droits de l'homme la protège encore d'une remise en cause frontale, même si des voix commencent à la déclarer obsolète.

2 Les principes essentiels du régime de Genève sont néanmoins aujourd'hui caducs. La notion même d'asile politique est mise en sourdine, l'asile en Europe n'est plus vu comme un bel outil de défense de la liberté mais comme un devoir humanitaire envers ceux qui réussissent à s'imposer sur son territoire. Le droit de quitter son pays, sacralisé dans la Déclaration universelle des droits de l'homme de 1948, est oublié au profit d'une nouvelle antienne: le droit de rester en sécurité dans son propre pays. L'asile dans les démocraties occidentales n'est plus au goût du jour, l'asile doit maintenant être recherché au plus près des lieux de conflits sous peine d'être considéré comme une migration économique.

Bien que la construction de l'Union européenne ne soit pas à l'origine de cette transformation du mode de protection des réfugiés, cette métamorphose s'est accomplie dans le cadre de l'harmonisation des procédures d'asile au sein de l'Union et aujourd'hui la politique d'asile européenne ne trouve plus sa source à Genève mais à Bruxelles. Ce régime de Bruxelles qui ne dit pas encore son nom est un mode de gestion de la protection des personnes persécutées apparemment fort différent du régime de Genève. Mais si les modalités d'actions changent, la logique d'action des États qui apportent leur protection reste constante. L'organisation du régime de Bruxelles répond aux mêmes règles que celle du régime de Genève. 


\section{Droits de l'homme et enjeux de la protection}

4 La problématique des droits de l'homme semble être le cadre naturel de l'analyse de l'organisation de l'asile. La possibilité de trouver refuge est un droit reconnu par la déclaration universelle des droits de l'homme de 1948: «1. Devant la persécution, toute personne a le droit de chercher asile et de bénéficier de l'asile en d'autres pays. 2. Ce droit ne peut être invoqué dans le cas de poursuites réellement fondées sur un crime de droit commun ou sur des agissements contraires aux buts et aux principes des Nations Unies" (article 14). L'asile est le dernier recours des réfugiés pour se voir reconnaitre les droits fondamentaux à la liberté et à la sécurité affirmés dans l'article 3 de la même déclaration : "Tout individu a droit à la vie, à la liberté et à la sûreté de sa personne ». Offrir l'asile est un devoir moral qui s'impose aux souverains depuis la nuit des temps; dans Les Suppliantes d'Eschyle, par exemple, le roi des Argiens, malgré les risques pour son peuple, ne peut refuser d'accueillir les Danaïdes sans renier ses dieux.

5 Malgré tout cela, la défense des droits de l'homme n'est pas le bon angle d'étude permettant de comprendre l'organisation de la protection des réfugiés. Sans s'attarder sur les relations de subordination entre le devoir moral et la raison d'État, une sélection opérée entre les droits de l'homme va nous mettre sur la voie d'une grille d'analyse plus pertinente. L'asile politique tel qu'il est conçu aujourd'hui ${ }^{1}$ protège le droit à la liberté et la sûreté, il ne protège pas le droit à la vie. En effet, tant dans la déclaration des droits de l'homme que dans la convention de Genève, les criminels, les anciens tortionnaires et autres tyrans déchus risquant à leur tour pour leur vie sont exclus du bénéfice de l'asile. De plus, l'asile est conçu pour protéger exclusivement contre la persécution, pas contre la famine même lorsque celle-ci est mortelle. Un demandeur d'asile qui n'évoquerait que le danger de mourir de faim dans son pays à l'appui de sa demande se verrait irrémédiablement débouté.

6 La protection contre les persécutions ne protège pas la vie en tant que telle mais l'humanité, dans le sens où cette protection s'oppose à la négation, inhérente au processus même de persécution, de l'appartenance des victimes à l'espèce humaine. L'asile protège le droit à une vie libre en société. Le droit à la vie des personnes en danger de famine n'est cependant pas oublié par la communauté internationale. Ces populations sont secourues par le Programme Alimentaire Mondial (PAM) de l'ONU comme les victimes de persécution le sont par le Haut Commissariat aux Réfugiés (HCR).

7 Les modes d'interventions du PAM et du HCR sont historiquement très différents, le premier, depuis sa création, intervient dans les pays d'origine, le second ne secourait jusqu'à très récemment les personnes persécutées qu'une fois qu'elles avaient fui leur pays. Ces choix résultent d'une apparente évidence, l'aide alimentaire est en général acceptée par les autorités locales et peut donc être apportée sur place; l'aide aux persécutés n'est jamais acceptée par les persécuteurs et, à moins d'intervenir militairement, la protection doit donc être organisée autrement.

8 C'est là une clef essentielle pour comprendre l'évolution de l'asile. Si la protection est organisée pour les réfugiés, elle est avant tout chose organisée par les États d'accueil en fonction des rapports de force internationaux. Toute protection garantie par un État est en effet une intervention dans un conflit, même si le paravent de la neutralité humanitaire est aujourd'hui de plus en plus souvent utilisé pour tenter de dissimuler cette réalité incontournable. La conjoncture des relations politiques internationales est 
ainsi de facto au cœur de l'organisation de la protection, mais elle n'y est pas seule, la conjoncture socio-économique est également très présente via les politiques migratoires puisque l'asile implique une immigration internationale.

9 La grille d'analyse pertinente pour comprendre les changements survenus dans le mode d'organisation de l'asile est donc beaucoup plus centrée sur les enjeux de la protection des réfugiés en terme de relations internationales et de politiques migratoires que sur le respect des droits de l'homme, les États actuels n'y étant ni plus ni moins sensibles que ceux d'hier. Ce qui n'empêche pas les droits de l'homme de jouer un rôle dans l'organisation de l'asile ; d'une part ils sont une référence obligée, bien que souvent purement formelle, pour toutes les démocraties, d'autre part ils sont souvent instrumentalisés (de même que les sentiments humanitaires). Une petite chronologie des évolutions du mode d'organisation de l'asile va nous permettre de montrer à quel point celui-ci est le reflet de son époque.

\section{1, l'instrumentalisation des droits de l'homme}

10 À la fin de la guerre, le rapatriement des réfugiés est confié à l'Administration des Nations Unies pour le secours et la reconstruction (UNRRA). Des rapatriements forcés de ressortissants soviétiques discréditent son action et cette institution est dissoute en 1947. L'Organisation internationale pour les réfugiés (OIR) est alors créée avec comme mission la réinstallation des réfugiés dans les pays tiers. «Cette politique permet à des pays européens en pleine reconstruction de bénéficier d'une main d'ouvre abondante. Elle est, à ce titre, dénoncée par les pays du bloc de l'Est" (Documentation Française 2004). Cette citation résume parfaitement l'intérêt économique des réfugiés pour les pays européens. Dès la fin des années quarante, les réfugiés fuyant les régimes communistes s'ajoutent aux réfugiés de la guerre et les Nations Unies, prenant conscience du caractère durable du problème, décident de se doter d'une nouvelle institution. Les statuts du HCR sont adoptés en décembre 1949 et la convention des Nations Unies relative au statut des réfugiés (dite convention de Genève) est adoptée à Genève en juillet 1951.

11 L'organisation de la protection des personnes persécutées mise en place en 1951 reflète ce contexte: l'intervention dans les pays d'origine est exclue, les besoins de maind'œuvre incitent à faire appel à l'immigration, les réfugiés qui réussissent à quitter le bloc de l'Est demandent l'asile. Le régime de Genève répond à cette demande et va même beaucoup plus loin. Avec la définition du réfugié de la convention de Genève, l'asile n'est pas humanitaire mais politique. Il ne protège pas la vie humaine en général ${ }^{2}$ mais la liberté, et plus précisément la liberté de ne pas être persécuté pour des raisons d'appartenance à une race, une nationalité, une religion, un groupe social, ou pour ses opinions politiques.

12 De plus ce régime stimule la demande d'asile en mettant l'accent sur le droit de partir consacré en 1948 dans la déclaration universelle des droits de l'homme: "1. Toute personne a le droit de circuler librement et de choisir sa résidence à l'intérieur d'un État. 2. Toute personne a le droit de quitter tout pays, y compris le sien, et de revenir dans son pays ». (article 13).

13 L'accueil des réfugiés au nom de la défense de la liberté a l'immense intérêt de mettre en évidence les négations des droits de l'homme dans les régimes communistes et de discréditer ces derniers. Dès les débuts de la guerre froide l'asile a ainsi un intérêt 
stratégique. Et il s'agit bien d'un intérêt stratégique et non d'un engagement fondamental pour la défense des droits humains puisqu'à la même époque le droit des peuples à disposer d'eux-mêmes n'est pas à l'ordre du jour dans les colonies des démocraties capitalistes. L'engagement au service de la liberté, manifeste dans la définition du réfugié, est de plus tempéré par le préambule de la convention de Genève qui affirme la neutralité de l'asile ${ }^{3}$, et par une absence remarquable : la convention est muette sur les procédures de reconnaissance de la qualité de réfugié et sur leur droit au séjour. En fonction du principe qu' «est maître des lieux celui qui les organise ${ }^{4}$ » les États ont toute latitude pour interpréter la convention et ainsi maîtriser les flux de réfugiés à leur guise. Précaution supplémentaire, dans sa rédaction initiale, la convention de Genève limite son champ d'application aux évènements survenus avant le $1^{\mathrm{er}}$ janvier 1951, les pays signataires pouvant de plus en option restreindre ce champ aux événements survenus en Europe.

\section{7, l'extension de la guerre froide à l'ensemble du monde et le protocole de New York}

14 La limitation temporelle initiale de la convention de Genève s'est vite avérée une précaution superflue. Le cadre de la convention de Genève était efficace et si on pouvait affirmer que tout événement dans le monde communiste, comme le soulèvement de Budapest et la fuite des réfugiés hongrois, avait des racines antérieures à 1951, il n'en était pas de même pour les évènements survenus hors d'Europe. La communauté internationale a donc décidé de lever les réserves de temps et d'espace de la convention de Genève par un protocole signé à New York en 1967 et ratifié par la France en 1971.

Ce protocole ne correspond pas à un soudain élan de générosité envers les réfugiés du tiers monde mais à une évolution de l'intérêt stratégique de l'asile. Dans le cadre de l'extension de la guerre froide à l'ensemble du monde, le contrôle des flux de réfugiés hors d'Europe devenait un enjeu considérable. Le HCR reconnaît aujourd'hui l'importance qu'allait prendre quelques années plus tard le contrôle des camps de réfugiés dans le conflit entre blocs. "Pendant les années soixante-dix et quatre-vingt [...] Si le gouvernement d'un pays du tiers monde était soutenu par l'une des deux superpuissances, l'autre essayait de renverser, ou au moins de saper ce régime en soutenant un mouvement rebelle, assez souvent, il faut bien l'admettre, sous couvert de programme d'assistance humanitaire et d'aide aux réfugiés » (HCR, 1997 : 22).

\section{9, un système d'asile spécial tiers-monde}

Avant même d'être ratifié par tous les États signataires, le protocole de New York avait en partie manqué son objectif affiché. En effet, s'il levait les réserves de temps et d'espace de la convention de Genève, il ne levait pas une ambiguïté originelle de la définition du réfugié : les réfugiés de guerre entrent-ils dans le cadre de la convention? Conçu en partie pour résoudre la question des réfugiés de la deuxième guerre mondiale on pourrait penser que oui. Cependant, les pays occidentaux souhaitant éviter la possibilité d'une arrivée massive sur leur sol et se référant à l'esprit et non à la lettre de la convention ont toujours estimé que le réfugié est une personne ayant des craintes 
individuelles de persécutions, ce qui exclut du champ de la convention les victimes de guerre et autres désordres généralisés.

17 Cette exclusion allant à l'encontre de la volonté de gérer dans le cadre général de l'asile les flux de réfugiés générés par les conflits par procuration entre les deux blocs, la difficulté a été résolue en élaborant des instruments régionaux : la convention de l'OUA en 1969 et plus tard la déclaration de Carthagène de 1984 identique dans son principe. La convention de l'OUA reprend la définition du réfugié de la convention de Genève et $\mathrm{y}$ ajoute un deuxième alinéa. "Le terme "réfugié" s'applique également à toute personne qui, $d u$ fait d'une agression, d'une occupation extérieure, d'une domination étrangère ou d'événements troublant gravement l'ordre public dans une partie ou dans la totalité de son pays d'origine ou du pays dont elle a la nationalité, est obligée de quitter sa résidence habituelle pour chercher refuge dans un autre endroit à l'extérieur de son pays d'origine ou du pays dont elle a la nationalité".

18 Ce choix d'élaborer des instruments régionaux permet au HCR de gérer les camps de réfugiés dans le tiers monde en toute légitimité et aux pays riches de maintenir leur interprétation restrictive destinée à les protéger de flux massifs éventuels.

\section{3-74, choc pétrolier et premiers intérêts contradictoires}

19 À la suite du choc pétrolier et de la crise économique de 1973, les démocraties européennes ont anticipé les conséquences sociales des restructurations industrielles prévues, en limitant l'immigration économique. En France, la fermeture des frontières de 1974 bloque le système des migrations tournantes, fixe la population étrangère sur place, et développe l'immigration clandestine. Cependant, l'opposition, entre l'accueil des demandeurs d'asile et la politique migratoire n'est pas immédiate. Au contraire, l'accueil des réfugiés en provenance du Chili après le coup d'État contre Salvador Allende (Vabre, 2004), puis celui des boat-people du Sud-Est asiatique après la prise de Saigon sont relativement généreux. Les boat-people qui fuient un régime communiste ont bonne presse, il faut dire que ces quotas implicites de réfugiés arrivent au tout début de l'arrêt de l'immigration économique non qualifiée et que certaines industries ont encore besoin d'un peu de temps pour s'adapter. Dès 1974 cependant, les réfugiés d'autres origines, comme ils étaient nommés dans les rapports d'activité de l'office Français pour la protection des Réfugiés et des Apatrides (OFPRA) sont suspectés de venir pour des raisons économiques et le taux de rejet de leurs demandes d'asile commence à augmenter.

Sur le plan international, les restrictions migratoires dans les pays riches ne remettent pas en cause le système de l'asile pour deux grandes raisons. D'une part, l'asile dans les pays riches n'est pas immédiatement utilisé pour contourner l'arrêt de l'immigration économique. En France, par exemple, les premiers détournements de la procédure d'asile à fin d'immigration économique ne sont statistiquement perceptibles qu'au milieu des années quatre-vingt soit dix ans après la fermeture des frontières. D'autre part, le milieu des années soixante-dix est la période de plein développement de l'opposition entre les deux blocs dans le tiers monde et nous avons vu l'intérêt militaire de la gestion des flux de réfugiés. 


\section{5, la libre circulation des personnes et l'accord de Schengen}

21 Le milieu des années quatre-vingt voit se développer la diversification des origines nationales des demandeurs d'asile, la lutte contre l'immigration clandestine, le transfert de la clandestinité vers la demande d'asile avec les détournements de procédures associés, et le "laboratoire Schengen». La libre circulation des ressortissants des pays signataires et le report des contrôles aux frontières communes marquent en Europe le début d'une harmonisation des politiques restrictives en matière d'asile, notamment en terme de renvoi des demandeurs dans le pays responsable de leur entrée sur le territoire commun. Cet accord, complété par la convention d'application de 1990, n'entrera formellement en vigueur qu'en 1995 mais ses effets se feront sentir dès 1985. En ce qui concerne la demande d'asile, «l'acquis Schengen " (c'est-à-dire la règle de la réadmission des demandeurs d'asile entre les États membres de l'Union en fonction du principe que toute personne doit pouvoir demander l'asile dans un pays de l'Union mais dans un seul et que le pays responsable du traitement de la demande est celui qui a pris la plus grande part dans l'entrée du demandeur d'asile sur le territoire commun), sera repris dans la convention de Dublin de 1990 entrée formellement en vigueur en 1997 puis remplacée en 2003 par le règlement européen dit Dublin II qui en peaufine l'application.

\section{9, la fin de l'intérêt stratégique de l'asile en Europe}

La chute du mur de Berlin est la date symbolique de la fin de la guerre froide. Avec le mur c'est le système soviétique qui s'est écroulé et avec lui l'intérêt stratégique de l'organisation de l'asile mise en place par le régime de Genève. L'intérêt économique de l'asile étant déjà perçu ${ }^{5}$ comme totalement négatif depuis de nombreuses années avec l'arrêt de l'immigration économique, il ne reste alors pour défendre l'asile en Europe que le drapeau des droits de l'homme. Ce drapeau auquel les démocraties occidentales sont très attachées a protégé jusqu'à aujourd'hui la convention de Genève. Celle-ci n'a pas été reniée formellement, par contre elle est marginalisée par le développement d'un nouveau droit européen. En 2003, le ministre des affaires étrangères français reconnaissait cette évolution et la trouvait même trop lente: "le phénomène de la multiplication des demandes infondées est européen [...] un nouveau droit s'élabore à Bruxelles ${ }^{6}$, mais nous devons agir sans attendre pour faire face à un afflux sans précédent $»^{7}$.

\section{Années quatre-vingt dix, la maîtrise des flux de réfugiés}

La mise en place de la maitrise des flux a commencé par les mesures destinées à réduire le nombre de demandes d'asile infondées. Au début des années quatre-vingt dix, dans les pays se réclamant des droits de l'homme la réduction n'était pas encore censée concerner les réfugiés statutaires. 

enchaînement de moyens que l'on peut résumer ainsi :

- - la dissuasion : tous les États cherchent à être moins attractifs que leurs voisins en compliquant la vie des demandeurs d'asile : délai d'enregistrement des demandes, interdiction de travailler, dispositifs d'hébergement sous-dimensionnés, etc.;

- - l'endiguement : obligation de visa pour les ressortissants des pays d'origine des demandeurs d'asile conjuguée à une obligation pour les entreprises de transports de vérifier les visas sous peine de lourdes amendes ( 5000 euros par personne transportée plus les frais de retour);

- - le refoulement : en vertu du principe que les demandeurs d'asile doivent déposer leur requête dans le premier pays démocratique qu'ils traversent, les accords de réadmission (Dublin II entre États membres et accords bilatéraux avec les pays voisins) permettent de renvoyer en cascade les demandeurs d'asile à la périphérie de l'Union lorsque ces derniers arrivent illégalement par voie terrestre (cas le plus fréquent de par la difficulté d'obtention des visas);

- le rejet de la demande : la procédure est discrétionnaire, légalement il n'est pas demandé de preuves de persécutions mais sans preuves irréfutables les récits des demandeurs ne sont pas jugés crédibles. La notion de demande « hors champ » de la convention de Genève est aussi couramment employée. En France et jusqu'en 2004, les victimes de persécutions non étatiques, victimes des groupes islamiques armés par exemple, étaient considérées comme n'entrant pas dans l'esprit de la convention de Genève. ceux du Sud rejettent les demandes et laissent les demandeurs dans une illégalité plus ou moins tolérée en attendant qu'ils aillent voir ailleurs. La France se situe dans le groupe du Sud. Une tentative de gérer les personnes dites hors champ de la convention de Genève mais ayant des craintes fondées en cas de renvoi, a été menée a partir de 1998 avec l'asile territorial créé par la loi Chevènement, mais elle a échoué. Cet asile, géré par les préfectures dont ce n'était manifestement pas la culture, n’a été offert qu'à $1,3 \%$ des demandeurs en cinq années d'existence.

Par anticipation à une directive européenne adoptée en 2004, la loi sur l'asile de 2003 a remplacé l'asile territorial par la protection subsidiaire gérée par l'OFPRA. La protection subsidiaire est le nouveau nom pour désigner la protection précaire harmonisée devant remplacer les multiples statuts humanitaires dans l'Union. En 2003 dans l'Europe des quinze, déjà moins de la moitié des réfugiés était protégée par la convention de Genève. Ce changement de mode de protection n'est pas anodin, la convention de Genève offre la possibilité d'une installation durable (en France, droit au séjour de dix ans automatiquement renouvelable) alors que la protection subsidiaire vise à empêcher ce type d'installation en n'accordant qu'un droit au séjour d'un an. Ce 
passage de l'asile politique à l'asile humanitaire est une conséquence directe de la fin de la guerre froide.

\section{7, la communautarisation de la politique d'asile}

L'entrée en vigueur le $1^{\mathrm{er}}$ mai 1999 du traité d'Amsterdam signé en 1997 marque le début du compte à rebours de la création d'un véritable régime communautaire de l'asile dans l'Union Européenne. Ce traité fixe un délai de cinq ans pour parfaire l'harmonisation des procédures d'asile au sein de l'Union avant que l'asile intègre le «premier pilier » dans le cadre duquel l'unanimité n'est pas requise pour les prises de décision au Conseil des ministres. Deux grandes directives sur l'asile, l'une sur les statuts à utiliser (convention de Genève et protection subsidiaire), l'autre sur les conditions de délivrance de ces statuts (asile interne, pays d'origine sûrs, pays tiers sûrs), en gestation difficile depuis plus de deux ans, ont été approuvées le 29 avril 2004, deux jours avant l'entrée dans l'Union de dix nouveaux pays.

\section{4, le régime de Bruxelles}

Le régime de Bruxelles n'est pas encore totalement finalisé mais ses grandes lignes sont déjà écrites. Comme le régime de Genève, il est parfaitement en phase avec son époque, belliqueuse est complexe.

- - Contrairement aux espérances, la fin de la guerre froide n'a pas mis fin aux multiples conflits sur la planète, bien au contraire, la désacralisation des frontières a ouvert la voie à de nouveaux conflits. La gestion des réfugiés est donc toujours un problème important qui se pose aux États européens mais les origines des demandeurs d'asile sont bien plus diversifiés qu'au lendemain de la Deuxième Guerre mondiale.

- - L'Europe est toujours très attachée au symbole des droits de l'homme, même si elle les met entre parenthèses lors des visites des présidents russes ou chinois si peu respectueux de ces droits.

- - L'Europe n'a plus besoin d'immigrés peu qualifiés et se protège d'eux au point d'être couramment désignée par l'expression « Europe forteresse ».

- L'Europe participe à la mondialisation des échanges et au maintien de l'ordre international nécessaire à cette globalisation économique.

La nouvelle organisation de l'asile répond à tous ces points au prix d'une certaine complexité. En dehors de l'illégalité tolérée qui en est le niveau zéro, on dénombre trois grands niveaux de protection : l'asile convention de Genève, la protection subsidiaire, l'asile sur place dans les pays d'origine.

Le statut de réfugié de la convention de Genève est conservé, mais n'est octroyé qu'avec une extrême parcimonie. Ceci a l'avantage de préserver l'image flatteuse d'une Europe terre des droits de l'homme sans conséquence en terme de flux migratoires. Le maintien de la convention de Genève est aussi caractéristique du passage très progressif du régime de Genève à celui de Bruxelles, sans bousculer les institutions et notamment le HCR si utile pour gérer l'asile sur place.

La protection subsidiaire, comme son nom l'indique, est une protection complémentaire à la protection principale, destinée aux demandeurs d'asile ayant subi des persécutions qui n'entrent pas dans le champ de cette dernière. Or le champ de la 
convention de Genève, censée être la protection principale, est très vaste puisqu'il couvre tous les risques de persécutions liés à la race, la religion, la nationalité, l'appartenance à un certain groupe social, et les opinions politiques. De plus, les persécutions non-étatiques ne sont plus hors-champ depuis la directive européenne du 29 avril 2004

La directive du 29 avril 2004 définit ainsi les personnes pouvant bénéficier de la protection subsidiaire : «tout ressortissant d'un pays tiers ou tout apatride qui ne peut être considéré comme un réfugié, mais pour lequel il y a des motifs sérieux et avérés de croire que la personne concernée, si elle était renvoyée dans son pays d'origine ou, dans le cas d'un apatride, dans le pays dans lequel il avait sa résidence habituelle, courrait un risque réel de subir les atteintes graves définies à l'article $15 \%$. Celui-ci définit ainsi ces atteintes graves :

«a) la peine de mort ou l'exécution;

b) la torture ou des traitements ou sanctions inhumains ou dégradants infligés à un

demandeur dans son pays d'origine ;

c) des menaces graves et individuelles contre la vie ou la personne d'un civil en

raison d'une violence aveugle ou en cas de conflit armé interne ou international ».

Dans les deux premiers alinéas ce texte définit le type de persécution et non les motifs, or bien rares doivent être les motifs n'entrant pas dans le champ de la convention de Genève . Le troisième alinéa est plus vague sur le type de persécution mais invoque des circonstances: la violence aveugle et les conflits armés internes ou internationaux. Nous voici bien proches du deuxième alinéa de la définition du réfugié de la convention de l'OUA déjà citée. Mais si la convention de l'OUA reconnait le statut de réfugié aux victimes des conflits armés et de la violence généralisée, la directive n'accorde qu'une protection précaire assortie d'un droit au séjour d'un an révocable et éventuellement renouvelable. De plus cette protection ne s'applique qu'en cas de menaces individuelles, ce qui semble peu compatible avec la notion de violence aveugle.

En fait, cette protection subsidiaire joue un rôle particulier. Elle n'est pas réellement conçue pour compléter la protection de la convention de Genève - les mesures d'endiguement et de refoulement n'auraient pas lieu d'être si on souhaitait protéger plus efficacement les demandeurs d'asile - mais pour attribuer un titre à ceux qui ont réussi à pénétrer dans la forteresse et que l'on ne sait pas expulser en raison des risques avérés qu'ils encourent dans leur pays. La délivrance de ce statut relève du maintien de l'ordre et ce n'est pas un hasard si les ancêtres de ce statut ont vu le jour dans les pays où l'informel est le moins toléré.

D'après les statistiques du HCR, les statuts humanitaires représentaient

déjà $46 \%$ des protections accordées en 2002 dans l'Europe des Quinze ; et, dans les pays précurseurs, Hollande et Suède, ce pourcentage était respectivement de $91 \%$ et $94 \%$.

L'asile sur place dans les pays d'origine est l'asile d'aujourd'hui. Il répond à la fois aux besoins de protection (partiellement), à la volonté de réduire les flux vers l'Europe, et à la nouvelle donne des relations internationales. Dans le cadre de la globalisation économique la position de gendarme du monde est un enjeu majeur et le droit d'ingérence, si longtemps occulté par le conflit Est-Ouest, est redécouvert aujourd'hui fort à propos par les puissances occidentales. Mais ce droit d'ingérence a besoin d'une justification pour ne pas apparaître comme l'expression de la seule force brute et les droits de l'homme sont à nouveau instrumentalisés. Le "droit de rester en sécurité dans son propre pays ", selon la phraséologie consacrée, est aujourd'hui reconnu comme un 
droit fondamental que les démocraties doivent défendre (comme hier le droit de partir). C'est là le nouvel et véritable intérêt stratégique de la protection des victimes de persécutions: justifier les interventions militaires des puissances occidentales lorsque leurs intérêts sont en jeu ${ }^{10}$. L'histoire récente nous montre en effet que l'usage $\mathrm{du}$ droit d'ingérence est sélectif. Selon le contexte géopolitique tous les peuples opprimés ne sont pas défendus avec la même vigueur, les Tchétchènes, par exemple, ne sont pas protégés comme l'ont été les Kosovars, et quel État se soucie du sort des Tibétains?

Les sanctuaires humanitaires n'ont pas fait la preuve de leur efficacité en terme de protection des personnes, notamment à Srebrenica ${ }^{11}$, mais la directive de l'Union européenne du 29 avril 2004 prévoit expressément que l'asile ne peut être accordé dans l'Union si des organisations internationales, telles que l'ONU ou l'OTAN apportent une protection sur place.

Le régime de Bruxelles est ainsi organisé pour limiter les flux individuels et apporter une protection collective au plus près des conflits. Cette organisation de la protection, qui pourrait être efficace si la communauté internationale s'en donnait les moyens, ne peut cependant couvrir tous les besoins. Il ne saurait être question d'intervenir dans tous les États qui violent les droits de l'homme d'une minorité ou de leurs opposants. Pour toutes ces personnes en danger le régime de Bruxelles est une régression, l'endiguement et le refoulement leur ferment la route de la liberté.

\section{Conclusion}

Les profonds changements et la complexification du régime de l'asile l'ont paradoxalement rendu plus intelligible. Le passage de la défense du droit de partir à celle du droit de rester en réaction aux bouleversements géopolitiques démontre que l'attachement à ces droits est purement conjoncturel. Ce ne sont pas les droits de l'homme qui façonnent l'organisation de l'asile mais au contraire, c'est l'organisation de l'asile résultant des rapports de force entre États et des politiques migratoires des pays d'accueil qui fixent quels droits de l'homme seront médiatisés.

La fonction politique du régime de Genève (discréditer le monde communiste) n'a été reconnue dans les ouvrages du HCR (1995 et 1997) qu'à la fin de ce régime, et seulement pour justifier le changement de problématique. La fonction politique actuelle de l'asile dans les enclaves humanitaires au plus près des conflits (conforter la position de gendarme du monde) n'est pas encore avouable, bien que ces deux fonctions soient finalement assez proches : il s'agit dans les deux cas d'affirmer la suprématie des pays d'accueil.

\section{BIBLIOGRAPHIE}

ALLAND Denis, TEITGEN-COLLY Catherine (2002) Traité du droit d'asile, PUF, 693 p. 
BOUTEILLET-PAQUET Daphné (2001) L'Europe et le droit d'asile : la politique d'asile européenne et ses conséquences sur les pays d'Europe centrale, l'Harmattan, $396 \mathrm{p}$.

HCR (Haut Commissariat des Nations Unies pour les Réfugiés) (1995) Les réfugiés dans le monde. En quête de solutions, La Découverte, $264 \mathrm{p}$.

HCR (Haut Commissariat des Nations Unies pour les Réfugiés) (1997) Les réfugiés dans le monde. Les personnes déplacées : l'urgence humanitaire, La Découverte, 300 p.

JULIEN-LAFERRIERE François (1999) L'Union européenne Schengen et la liberté de circulation, in Philippe Dewitte (ed.) Immigration et intégration l'état des savoir, La Découverte, pp. 366-376.

LA DOCUMENTATION FRANÇAISE (2004), Les organisations en charge des réfugiés, Réfugiés et droits d'asile dans le monde, Dossiers d'actualité, avril 2004, consultable sur le site www ladocfrancaise.gouv.fr

LEGOUX Luc (2000) L'instrumentalisation de l'humanitaire dans la gestion de l'asile, Transeuropéennes, pp. 77-86.

LEGOUX Luc (2003) Nouvelle donne mondiale, nouvel asile, in Michelle Guillon, Luc Legoux, Emmanuel Ma Mung (eds), L'asile politique entre deux chaises, L'Harmattan, 2003, pp. 13-24.

MARIE Claude-Valentin (2002) Les politiques européennes de gestion des flux : contrôle et restrictions, Cahiers Français, $\mathrm{n}^{\circ} 307$ mars avril, pp. 57-65

VABRE Frédéric (2004) L'engagement comme ressource facilitant l'intégration : le cas des réfugiés latino-américains en Suède, Revue Européenne des Migrations Internationales, 2004.

\section{NOTES}

1. L'asile des sanctuaires existant jusqu'au $16^{\text {ème }}$ siècle avait, lui, une fonction de protection immédiate de la vie, en attendant qu'une solution soit trouvée au conflit considéré.

2. Voir les clauses d'exclusion déjà citées.

3. "Exprimant le vœu que tous les États, reconnaissant le caractère social et humanitaire du problème des réfugiés, fassent tout ce qui est en leur pouvoir pour éviter que ce problème ne devienne une cause de tension entre États » alinéas 5 du préambule de la convention de Genève.

4. Jean de la Fontaine.

5. L'asile est actuellement perçu comme un devoir coûteux, mais si on intègre les retombées positives pour les pays d'accueil il n'est pas certain que l'accueil des réfugiés n'ait aucun intérêt économique. Par exemple, la place des entreprises allemandes dans la reconstruction des pays issus de l'ex-Yougoslavie n'est certainement pas étrangère à leur accueil des réfugiés yougoslaves, mais on manque de connaissances quantitatives fines sur le sujet.

6. C'est nous qui soulignons.

7. Examen du projet de loi sur l'asile au Sénat, le 23 octobre 2003.

8. Et si l'harmonisation juridique est aujourd'hui acquise, l'harmonisation des pratiques est encore loin d'être faite.

9. À part la torture dans les cas de délits de droit commun, mais, bien que le risque de torture soit avéré dans certaines prisons russes ou turques, il est peu probable que des criminels de droit commun puissent obtenir l'asile pour cette raison.

10. Il faut noter que la justification humanitaire de l'ingérence est bien fragile puisque l'humanitaire est par définition neutre.

11. Où plus de 8000 personnes furent massacrées sous les yeux des casques bleus de l'ONU et 15 000 autres déportées avant d'être assassinées. 


\section{RÉSUMÉS}

Le régime de protection des réfugiés mis en place par la convention de Genève de 1951 a cédé la place à un nouveau régime de l'asile qui trouve sa source à Bruxelles. Le premier, centré sur le droit de quitter son pays, utilisait la défense des droits de l'homme et l'accueil des dissidents pour discréditer le monde communiste. Le second est plus complexe. Le statut de réfugié de la convention de Genève est reconnu à dose homéopathique; la protection subsidiaire permet d'encadrer les demandeurs d'asile que l'on sait débouter mais que l'on ne sait pas expulser; l'asile au plus près des zones de conflit permet de limiter les flux et de justifier le droit d'ingérence des puissances occidentales. L'évolution entre les deux régimes dévoile que ce sont les relations internationales et les politiques migratoires qui conditionnent l'organisation de l'asile et non les droits de l'homme.

Change and Continuity in the Protection of Refugees. The regime of protection for refugees put into place by the Geneva Convention of 1951 has given way to a new regime on asylum originating in Brussels. The first, centred around on the right to leave one's country, used the defence of human rights and the reception of dissidents to discredit the communist world. The second is more complex. Refugee status granted by the Geneva Convention is recognised in small doses; the subsidiary protection allows the supervision of the asylum seekers that can be dismissed but not expulsed; asylum near conflict zones helps to limit flows and to justify the right of Western powers to interfere. The evolution between the two regimes reveals that it is international relations and migration policy, and not human rights that condition the organisation of asylum.

Cambios y permanencias en la protección de los refugiados. El régimen de protección de los refugiados establecido por la Convención de Ginebra de 1951 ha dado paso a un nuevo régimen de asilo, originario de Bruselas. El primero se centra en el derecho a abandonar su país y utiliza la defensa de los Derechos Humanos y la acogida de disidentes para desacreditar al mundo comunista. El segundo, más complejo, reconoce a dosis homeopáticas el estatuto del refugiado de la Convención de Ginebra : la protección subsidiaria permite tener en cuenta a los demandantes cuyo asilo ha sido denegado pero que no pueden ser expulsados; en cuanto al asilo próximo a las zonas de conflicto, permite limitar los flujos y justificar el derecho de ingerencia de las potencias occidentales. La evolución entre los dos regímenes demuestra que son las relaciones internacionales y las políticas migratorias las que condicionan la organización del asilo y no los Derechos Humanos.

\section{INDEX}

Mots-clés : accueil, asile politique, politiques migratoires, protection juridique, réfugiés

\section{AUTEUR}

\section{LUC LEGOUX}

Démographe, Maître de Conférence Un Paris I, Institut de démographie, 90 rue de Tolbiac, 75013 Paris, e-mail : legoux@univ-Paris1.fr 